\title{
Modulation of Airway Epithelial Innate Immunity and Wound Repair by M(GM-CSF) and M(M-CSF) Macrophages
}

\author{
Sander van Riet ${ }^{a} \quad$ Annemarie van Schadewijk ${ }^{a} \quad$ Steve de Vos $^{b}$ \\ Nick Vandeghinste ${ }^{b}$ Robbert J. Rottier ${ }^{c}$ Jan Stolk ${ }^{a}$ Pieter S. Hiemstra ${ }^{a}$ \\ Padmini Khedoe ${ }^{a}$ \\ ${ }^{a}$ Department of Pulmonology, Leiden University Medical Center, Leiden, The Netherlands; ${ }^{b}$ Galapagos NV, \\ Mechelen, Belgium; ' Department of Pediatric Surgery, Erasmus MC-Sophia Children's Hospital, Rotterdam, \\ The Netherlands
}

\section{Keywords}

Macrophages · Wound repair · Airway epithelial cells · LL-37 · Coculture

\begin{abstract}
Airway epithelial cells and macrophages participate in inflammatory responses to external noxious stimuli, which can cause epithelial injury. Upon injury, epithelial cells and macrophages act in concert to ensure rapid restoration of epithelial integrity. The nature of the interactions between these cell types during epithelial repair is incompletely understood. We used an in vitro human coculture model of primary bronchial epithelial cells cultured at the air-liquid interface (ALI-PBEC) and polarized primary monocyte-derived macrophages. Using this coculture, we studied the contribution of macrophages to epithelial innate immunity, wound healing capacity, and epithelial exposure to whole cigarette smoke (WCS). Coculture of ALI-PBEC with lipopolysaccharide (LPS)-activated M(GM-CSF) macrophages increased the expression of DEFB4A, CXCL8, and IL6 at $24 \mathrm{~h}$ in the ALI-PBEC, whereas LPS-activated M(M-CSF) macrophages only increased epithelial IL6 expression. Furthermore, wound repair was accelerated by coculture with both activated M(GM-
\end{abstract}

\begin{tabular}{ll}
\hline karger@karger.com & (C) 2020 The Author(s) \\
www.karger.com/jin & Published by S. Karger AG, Basel Karger \\
& This article is licensed under the Creative Commons Attribution- \\
Karger & NonCommercial-NoDerivatives 4.0 International License (CC BY- \\
NC-ND) (http://www.karger.com/Services/OpenAccessLicense). \\
Usage and distribution for commercial purposes as well as any dis- \\
tribution of modified material requires written permission.
\end{tabular}

CSF) and M(M-CSF) macrophages, also following WCS exposure. Coculture of ALI-PBEC and M(GM-CSF) macrophages resulted in increased CAMP expression in $\mathrm{M}(\mathrm{GM}-\mathrm{CSF})$ macrophages, which was absent in M(M-CSF) macrophages. CAMP encodes LL-37, an antimicrobial peptide with immune-modulating and repair-enhancing activities. In conclusion, dynamic crosstalk between ALI-PBEC and macrophages enhances epithelial innate immunity and wound repair, even upon concomitant cigarette smoke exposure.

(C) 2020 The Author(s)

Published by S. Karger AG, Basel

\section{Introduction}

Airway epithelial cells play a central role in the first line of defense against inhaled particles, gasses, and pathogens. The epithelial lining acts as a physical barrier and epithelial cells produce protective mediators (e.g., cytokines, chemokines, antimicrobial peptides) to prevent intrusion of harmful substances and pathogens into the lungs. Epithelial cells also mediate mucociliary clearance to remove mucus-trapped particles and pathogens from the airways $[1,2]$. Injury to the epithelial layer, due to e.g. bacterial and/or viral infection or inhalation of toxicants 
(including cigarette smoke), may cause disruption of epithelial barrier integrity and impair epithelial repair $[1,3]$. The epithelial repair process is tightly controlled to ensure rapid closure of the wound and restoration of lung tissue homeostasis. However, chronic insults to the epithelial layer contribute to dysfunction of airway epithelial cells and development and progression of lung diseases, such as chronic obstructive pulmonary disease, idiopathic pulmonary fibrosis, and asthma. Epithelial integrity, barrier function, and host defense responses are impaired in various lung diseases [1,4], predisposing these patients to repeated infections and exacerbations. Inflammatory cells such as macrophages contribute to the epithelial wound repair process by releasing a range of mediators and by providing protection against infections following disruption of the epithelial barrier integrity $[5,6]$.

Macrophages constitute a heterogeneous population of cells resulting from their high level of plasticity, and the various subsets contribute to the epithelial repair response and host defense $[7,8]$. The phenotype of macrophages is tightly controlled by their microenvironment that provides signals for activation and differentiation. Insight into these mechanisms has resulted in a classification of macrophages based on their activation state and properties. Macrophages can thus be broadly subdivided into proinflammatory macrophages (known as classically activated macrophages or M1 macrophages) and anti-inflammatory macrophages (known as alternatively activated macrophages or M2 macrophages) $[9,10]$. Proinflammatory macrophages produce proinflammatory cytokines and their phenotype is driven by proinflammatory stimuli, including TNF- $\alpha$, IFN- $\gamma$, and lipopolysaccharide (LPS), whereas anti-inflammatory macrophages are more diverse and can be divided into several subsets which are involved in the defense against parasitic infections (M2a), immunoregulation (M2b), and tissue remodeling and matrix deposition (M2c) [11]. In the lungs, macrophages are widely present in the airway lumen (airway macrophages), the alveolar lumen (alveolar macrophages), and the lung parenchyma and airway wall (interstitial macrophages), whereas monocytes can be recruited upon inflammation [12]. The function and phenotype of these cells depends on the local cytokine milieu $[11,13]$.

Following injury to the lung epithelial lining, both resident macrophages and those derived from recruited monocytes contribute to the inflammatory and remodeling phase of epithelial repair, although the precise interaction with airway epithelial cells is insufficiently studied.

Airway Epithelial Cell Interaction with Macrophages
Despite the knowledge gained from various in vivo models on epithelial repair, the use of laboratory animals becomes more controversial and importantly the translation of results from such animal models to human disease is not always straightforward. However, whereas in vitro models with (primary) airway epithelial cells have provided much knowledge on the mechanism of epithelial wound repair $[5,14]$, these models do not accurately represent the complex cellular network of airway epithelial cells and inflammatory cells, including macrophages, that are essential during epithelial wound repair. Although various models are available to investigate the interaction between airway epithelial cells and immune and inflammatory cells, many of these studies have been performed using cell lines for either macrophages, airway epithelial cells, or both, and are therefore not representative. Furthermore, many of these models lack lung specificity. In the present study, we therefore combined primary airway epithelial cells with primary monocyte-derived macrophages. To study the complex cellular crosstalk and interaction between airway epithelial cells and macrophages in more detail, we developed a coculture model of human bronchial epithelial cells cultured at the air-liquid interface (ALI-PBEC) and human peripheral blood CD14+ monocyte-derived macrophages that were polarized to either a proinflammatory $\mathrm{M}$ (GM-CSF) or an anti-inflammatory M(M-CSF) macrophage phenotype [15]. Using this model of primary cells, we studied the interaction between $\mathrm{M}(\mathrm{GM}-\mathrm{CSF})$ or $\mathrm{M}(\mathrm{M}-\mathrm{CSF})$ macrophages and ALI-PBEC and its effect on epithelial innate immunity and repair.

\section{Materials and Methods}

Culture of Primary Bronchial Epithelial Cells

Primary bronchial epithelial cells (PBEC) were obtained from tumor-free lung tissue of patients undergoing lobectomy for lung cancer at the Leiden University Medical Center (Leiden, The Netherlands). All PBEC donors used for these experiments were considered not to have chronic airflow limitation (i.e., not to have chronic obstructive pulmonary disease), based on a predicted forced expiratory volume in $1 \mathrm{~s}>85 \%$, and all were aged $>55$ years at the time of surgery. The cells were isolated, cultured, and differentiated at the air-liquid interface (ALI) for 14 days (online suppl. Fig. 1A; see www.karger.com/doi/10.1159/000506833 for all online suppl. material) to develop a well-differentiated epithelial layer, in Transwell inserts in 12-well plates as previously described [16]. During PBEC differentiation, the cells were cultured at ALI with Bronchial Epithelial Cell Medium - basal (ScienCell, Carlsbad, CA, USA) diluted 1:1 with DMEM from Stemcell Technologies (Vancouver, BC, Canada) with bronchial epithelial cell growth supplements from ScienCell, further supplemented with the 
Table 1. Compounds used for mechanistic experiments

\begin{tabular}{lll}
\hline Compound & Concentration & Supplier \\
\hline LL-37 & $2.5 \mu \mathrm{g} / \mathrm{mL}$ & {$[40]$} \\
TGF- $\beta 1$ & $5 \mathrm{ng} / \mathrm{mL}$ & R\&D Systems \\
Anti-LL-37 (clone III D7) & & \\
$\quad(09 / 02 / 00$ ST) & $1: 100$ & {$[40]$} \\
GM6001 & $25 \mu \mathrm{M}$ & Merck \\
SB-431542 & $5 \mu \mathrm{M}$ & Sigma \\
\hline
\end{tabular}

50-nM synthetic retinoic acid analog EC23 (Tocris, Bio-Techne Ltd., Abingdon, UK). Well-differentiated ALI-PBEC were used for further coculture experiments. Approximately $1 \times 10^{6}$ ALI-PBEC were present on these inserts at the time of the experiment.

Isolation of Monocytes and Differentiation towards $M(G M-C S F)$ and $M(M-C S F)$ Macrophage Phenotype

CD14-positive monocytes were isolated from fresh buffy coats (Sanquin Blood Bank, Leiden, The Netherlands) obtained from healthy controls as described previously [15]. We seeded $0.5 \times 10^{6}$ monocytes per well of a 12 -well plate with either $5 \mathrm{ng} / \mathrm{mL}$ GM-CSF (R\&D Systems, Minneapolis, MN, USA) or $50 \mathrm{ng} / \mathrm{mL}$ M-CSF (Miltenyi Biotec, Auburn, CA, USA) to induce polarization to $\mathrm{M}$ (GM-CSF) or M(M-CSF) macrophages, respectively. Following 7 days of culture in RPMI 1640 medium (Invitrogen, Breda Life Technologies, The Netherlands) containing 10\% FCS (Invitrogen), $2 \mathrm{mM}$ L-glutamine, $100 \mathrm{U} / \mathrm{mL}$ penicillin, and $100 \mu \mathrm{g} / \mathrm{mL}$ streptomycin (all from Bio Whittaker, Walkersville, MD, USA), $\mathrm{M}$ (GM-CSF) or M(M-CSF) macrophages were stimulated with 100 ng/mL LPS (from Pseudomonas aeruginosa; Sigma-Aldrich, St. Louis, MO, USA) during coculture experiments (experimental outline shown in online suppl. Fig. 1A). After 7 days, before start of coculture with ALI-PBEC, similar numbers of M(GM-CSF) and $\mathrm{M}(\mathrm{M}-\mathrm{CSF})$ were counted $\left(\sim 3.2 \times 10^{6}\right.$ cells/well $)$. M(GM-CSF $)$ or $\mathrm{M}(\mathrm{M}-\mathrm{CSF})$ macrophages were characterized by high expression of CHI3L1 and IL-12/p40 release (M[GM-CSF]) or CD163 expression and IL-10 release (M[M-CSF]), respectively (online suppl. Fig. 2).

\section{Coculture of $M(G M-C S F)$ or $M(M-C S F)$ Macrophages and} ALI-PBEC

ALI-PBEC were cultured as described above. Twenty-four hours before coculture, the medium of ALI-PBEC was switched to epithelial culture medium [16] without growth factors, hydrocortisone, and EC23 (starvation medium, $24 \mathrm{~h}$ starvation). Coculturing was performed by transfer of the Transwell inserts seeded with ALI-PBEC to another 12-well plate that contained the polarized macrophages. All coculture experiment were performed in starvation medium with or without LPS in the basal compartment, for activation of macrophages. ALI-PBEC were (1) cocultured with (LPS-activated) M(GM-CSF) or M(M-CSF) macrophages for $24 \mathrm{~h}$ (online suppl. Fig. 1A), (2) mechanically wounded and then cocultured with LPS-activated M(GM-CSF) or M(M-CSF) macrophages until wound closure, or (3) mechanically wounded, exposed to whole cigarette smoke (WCS), and subsequently cocultured with LPS-activated M(GM-CSF) or M(M-CSF) macrophages until wound closure. Epithelial wounding was performed as de- scribed previously [3]. A list of compounds that were used for mechanistic experiments is shown in Table 1. These compounds were added during coculture of ALI-PBEC and M(GM$\mathrm{CSF}) / \mathrm{M}(\mathrm{M}-\mathrm{CSF})$ macrophages.

\section{WCS Exposure}

WCS exposure was performed as described previously [16]. In brief, well-differentiated ALI-PBEC cultures were placed in a modified hypoxic chamber for WCS or air (control) exposure. In these chambers, the cultures were exposed to either 4-5 min of cigarette smoke from 1 3R4F research cigarette (University of Kentucky, Lexington, KY, USA) or room air as control. Following 4-5 min of WCS exposure, the chambers were ventilated for $10 \mathrm{~min}$ to remove smoke from the chambers. The Transwell inserts were subsequently removed from the plate and transferred to the 12 -well plates containing the macrophages for coculture and placed back at $37^{\circ} \mathrm{C}$.

\section{Quantitative RT-PCR}

RNA was isolated from ALI-PBEC (from Transwell insert) and macrophages (from 12-well plate at bottom), separately, according to the manufacturer's instruction using Maxwell RNA extraction kits (Promega, Madison, WI, USA). Quantitative RT-PCR was performed as described previously [16] using the primer pairs listed in Table 2. qPCR reactions were performed in triplicate, corrected for the geometric mean of expression of two reference genes (ATP5B and RPL13A) selected using the NormFinder algorithm software [17]. Expression values were determined by the relative gene expression of a standard curve as determined by the CFX manager software and expressed as fold increase (Bio-Rad).

\section{ELISA}

Levels of the interleukins, IL-8 (R\&D Systems), IL-10 and IL12/p70 (BD Bioscience), and human beta-defensin 2 (hBD-2) (Antigenix America) were determined in supernatant, basal medium (IL-10, IL-12/p40, IL-8, hBD-2) or in apical wash (IL-8, hBD-2) according to the manufacturer's instructions.

\section{Apical Wash}

To assess the levels of mediators secreted to the apical side by well-differentiated ALI-PBEC, apical washes were collected as described [18]. Briefly, apical washes were performed by applying $100 \mu \mathrm{L}$ PBS for $10 \mathrm{~min}$. After $10 \mathrm{~min}$ the fluid was collected and stored at $-80^{\circ} \mathrm{C}$ pending analysis by ELISA as described above.

\section{Statistical Analyses}

The statistical significance of differences was assessed using one-way ANOVA or repeated-measures analysis followed by post hoc analysis using Fisher's least significant difference multiple comparison test using GraphPad Prism 7. Differences at $p<0.05$ were regarded as statistically significant.

\section{Results}

\section{LPS-Activated M(GM-CSF) and M(M-CSF)}

Macrophages Alter Epithelial Innate Immunity

As host defense is one of the key functions of airway epithelial cells, we first aimed to establish whether there 
Table 2. Primers used for RT-PCR

\begin{tabular}{lll}
\hline Gene & Forward primer sequence $\left(5^{\prime}\right.$ to $\left.3^{\prime}\right)$ & Reverse primer sequence $\left(3^{\prime}\right.$ to $\left.5^{\prime}\right)$ \\
\hline$A T P 5 B$ & TCACCCAGGCTGGTTCAGA & AGTGGCCAGGGTAGGCTGAT \\
RPL13A & AAGGTGGTGGTCGTACGCTGTG & CGGGAAGGGTTGGTGTCATCC \\
$C A M P$ & TCATTGCCCAGGTCCTCAG & TCCCCATACACCGCTTCAC \\
$C X C L 8$ & CTGGACCCCAAGGAAAAC & TGGCAACCCTACAACAGAC \\
$I L 6$ & CAGAGCTGTGCAGATGAGTACA & GATGAGTTGTCATGTCCTGCA \\
$C D 163$ & TTTGTCAACTTGAGTCCCTTCAC & TCCCGCTACACTTGTTTCAC \\
$C H I L 1$ & CTGTGGGGATAGTGAGGCAT & CTTGCCAAAATGGTGTCCTT \\
$H G F$ & TCCAGAGGTACGCTACGAAGTCT & CCCATTGCAGGTCATGCAT \\
$M M P 9$ & ACCTCGAACTTTGACAGCGAC & GAGGAATGATCTAAGCCCAGC \\
$P D G F A$ & CACCACCGCAGCGTCAA & CCTCACCTGGACTTCTTTTAATTTG \\
TGFB1 & CTAATGGTGGAAACCCACAACG & TATCGCCAGGAATTGTTGCTG \\
$I L E F B A$ & ATCAGCCATGAGGGTCTTG & GCAGCATTTTGTTCCAGG \\
\hline
\end{tabular}

was an effect of macrophages on epithelial host defense in our coculture model. To this end we used well-differentiated primary ALI-PBEC in the presence or absence of LPS-stimulated M(GM-CSF) or M(M-CSF) macrophages (online suppl. Fig. 1A). Following $24 \mathrm{~h}$ of coculture we measured the epithelial expression of host defense mediators (IL6,IL8, and DEFB4A). In the absence of LPS, we found no effect of M(GM-CSF) or M(MCSF) macrophages on the epithelial mRNA expression of $I L 6, I L 8$, or DEFB4A. In the presence of LPS, expression of IL6 mRNA was increased in ALI-PBEC upon coculture with both macrophage subtypes (Fig. 1a). Expression of CXCL8 was increased in ALI-PBEC upon coculture with LPS-activated M(GM-CSF) but not M(M-CSF) macrophages. Furthermore, epithelial expression of $D E F B 4 A$, the gene encoding $\mathrm{hBD}-2$, was increased upon coculture with LPS-activated macrophages, and this effect was significantly higher in coculture with $\mathrm{M}(\mathrm{GM}-\mathrm{CSF})$ compared to $\mathrm{M}(\mathrm{M}-\mathrm{CSF})$ macrophages (Fig. 1a). Expression of other host defense proteins in ALI-PBEC (CAMP, RNASE7) was not altered (data not shown). We further investigated this effect on ALI-PBEC innate immune responses at the protein level (Fig. 1b). Confirming our findings on gene expression level in ALI-PBEC, IL-8 was found to be increased in the basal medium of cocultures with LPSactivated macrophages but not in the unstimulated controls. No increased IL-8 levels were observed in the apical washes. hBD-2 was not detected in the basal medium (data not shown), but was secreted on the apical side of the ALI-PBEC cocultured with activated M(GMCSF) but not M(M-CSF) macrophages. Since airway epithelial cells do not respond to LPS (Fig. 1a, b) [19], these findings show that ALI-PBEC initiate host defense responses in coculture with LPS-activated M(GM$\mathrm{CSF}$ ) or M(M-CSF) macrophages.

\section{Both Activated M(GM-CSF) and M(M-CSF)}

Macrophages Enhance Epithelial Wound Repair

After demonstrating that LPS-activated macrophages can modulate epithelial host defense responses, we next continued by investigating whether LPS-activated $\mathrm{M}(\mathrm{GM}-\mathrm{CSF})$ or M(M-CSF) macrophages can alter epithelial wound repair. To this end, circular wounds were mechanically created in the epithelial layer [3] and subsequently cocultured with activated macrophages (online suppl. Fig. 1A, B). LPS alone in the absence of macrophages did not affect epithelial wound closure. However, coculture with both $\mathrm{M}$ (GM-CSF) and M(M-CSF) macrophages significantly increased epithelial wound closure compared to epithelial monocultures. Complete wound closure was reached 30 and $50 \mathrm{~h}$ after mechanical wounding in the presence of activated M(GM-CSF) or M(MCSF) macrophages, respectively, whereas monocultures reached only $75 \%$ at this time point, indicating that epithelial wound closure was enhanced consistently in cocultures with both LPS-activated M(GM-CSF) and M(MCSF) macrophages.

Since we previously reported that exposure to WCS decreases epithelial wound closure $[3,20]$, we investigated whether LPS-activated macrophages also enhanced epithelial wound closure in WCS-exposed ALI-PBEC cultures. To this end, ALI-PBEC were exposed to WCS following wounding and subsequently cocultured with 

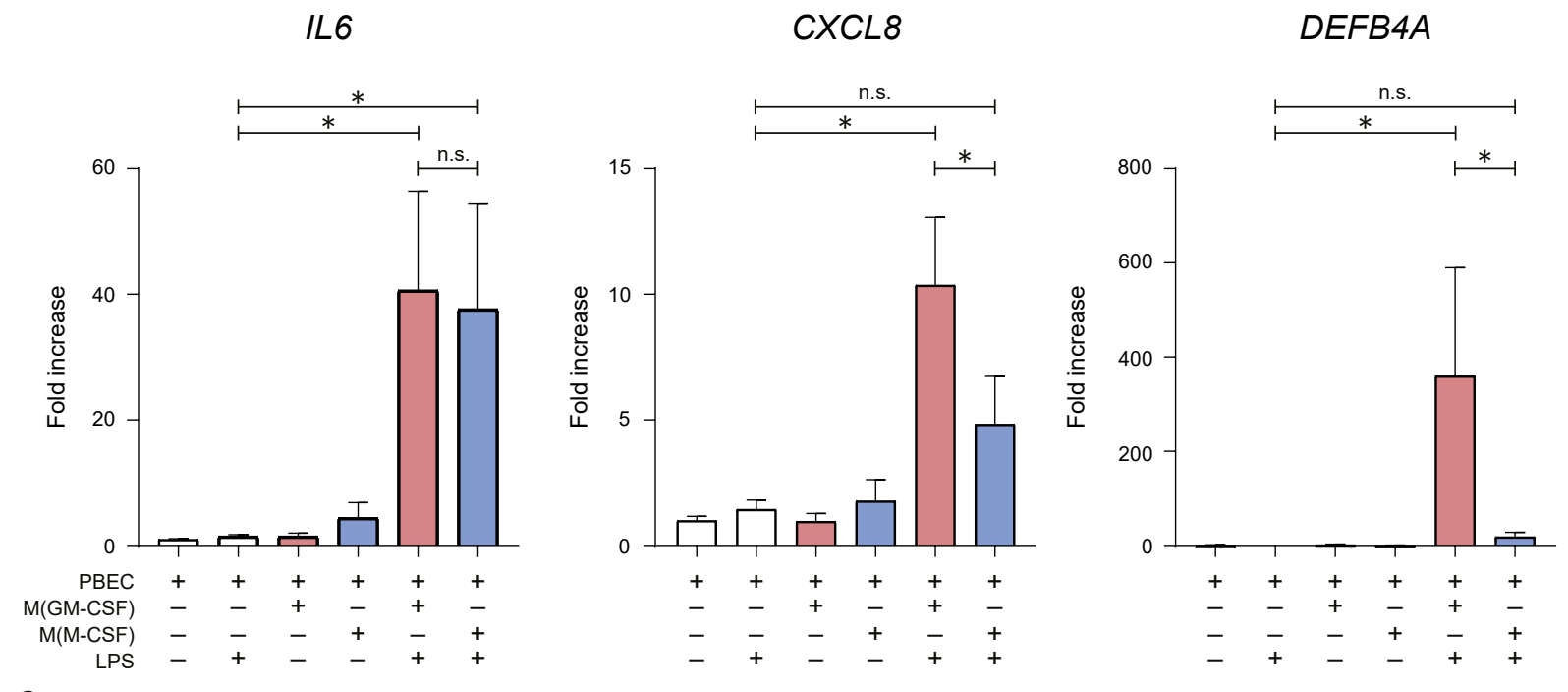

\section{IL-8 apical wash}

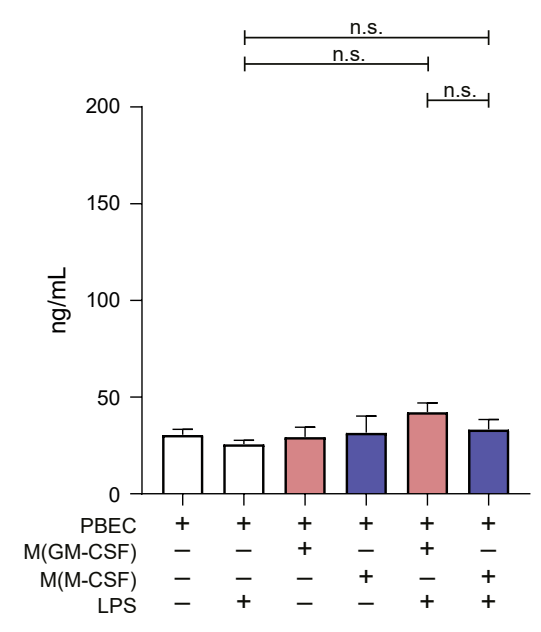

IL-8 basal medium

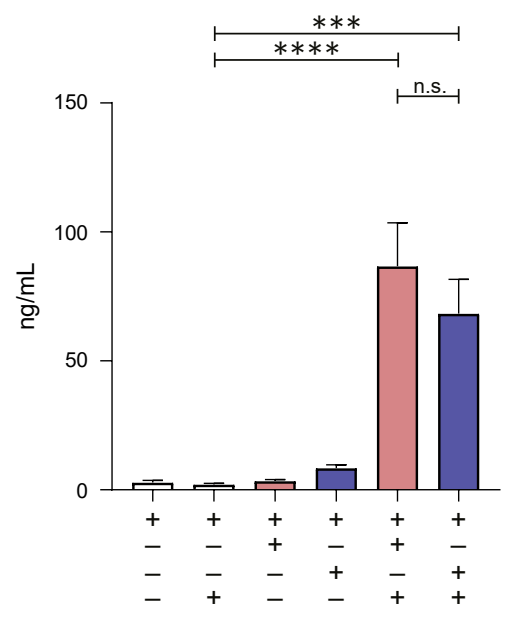

hBD-2 apical wash

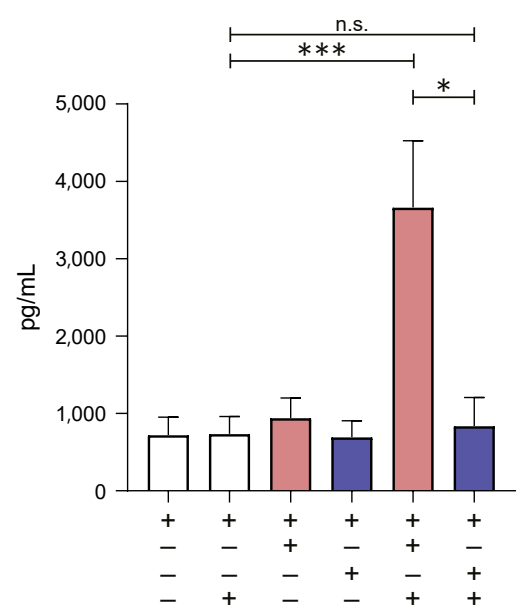

b

Fig. 1. Activated M(GM-CSF) and M(M-CSF) macrophages modulate epithelial innate immunity. Well-differentiated ALI-PBEC were cocultured with $\mathrm{M}(\mathrm{GM}-\mathrm{CSF})$ or $\mathrm{M}(\mathrm{M}-\mathrm{CSF})$ macrophages in the presence and absence of LPS, and mRNA and protein levels of cytokines and antimicrobial peptides were measured after $24 \mathrm{~h}$ in ALI-PBEC. a mRNA expression levels of $I L-6, C X C L 8$, and DEFB4A were measured in ALI-PBEC upon coculture with (activated) M(GM-CSF) (red bars) and M(M-CSF) (blue bars) macro- phages ( 7 independent ALI-PBEC donors). b IL- 8 and hBD-2 protein levels were measured at $24 \mathrm{~h}$ in basal medium and apical washes (7 independent ALI-PBEC donors). Data are shown as mean \pm SEM. ${ }^{*} p<0.05,{ }^{* * *} p<0.001,{ }^{* * * *} p<0.0001$. ALI-PBEC, primary bronchial epithelial cells cultured at the air-liquid interface; hBD2 , human beta-defensin 2; LPS, lipopolysaccharide; PBEC, primary bronchial epithelial cells. either M(GM-CSF) or M(M-CSF) macrophages (Fig. 2b). In line with our previous findings [14], WCS exposure delayed wound closure especially at early time points $(t=8 \mathrm{~h} ; p=0.065)$, irrespective of co- or monoculture of ALI-PBEC. Coculture with both M(GM-CSF) and M(M-
CSF) macrophages also significantly increased epithelial wound closure in WCS-exposed cultures. These data suggest that LPS-activated macrophages increase epithelial wound repair, also following exposure to cigarette smoke. 


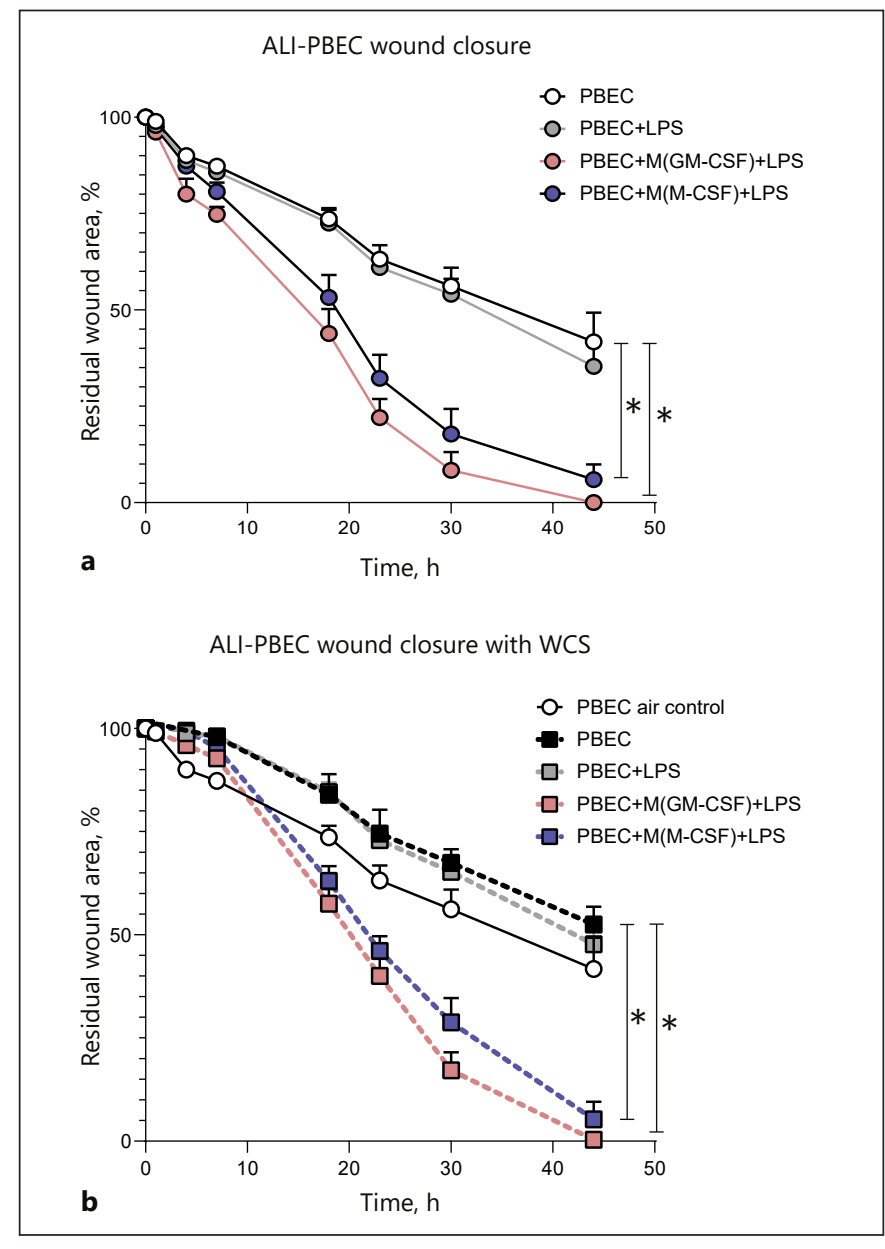

Fig. 2. Activated $M(G M-C S F)$ and $M(M-C S F)$ macrophages enhance epithelial wound repair. ALI-PBEC were mechanically injured and subsequently cocultured with either activated M(GM-CSF) or M(MCSF) macrophages. Wound closure was monitored in time using phase-contrast light microscopy. Wound closure is shown as percentage residual wound area. a Upon mechanical wounding, ALIPBEC were cultured alone (black line) or cocultured with activated $\mathrm{M}(\mathrm{GM}-\mathrm{CSF})$ (red line) or M(M-CSF) (blue line) macrophages (4 independent ALI-PBEC donors). $\mathbf{b}$ ALI-PBEC were exposed to WCS and subsequently cocultured with activated M(GM-CSF) (red line) or M(M-CSF) (blue line) macrophages (7 independent ALI-PBEC donors). Data are shown as mean \pm SEM. ${ }^{*} p<0.05$. ALI-PBEC, primary bronchial epithelial cells cultured at the air-liquid interface; LPS, lipopolysaccharide; PBEC, primary bronchial epithelial cells; WCS, whole cigarette smoke.

Fig. 3. Macrophage-derived mediators enhance epithelial wound repair. mRNA expression levels of various genes in M(GM-CSF) and $\mathrm{M}(\mathrm{M}-\mathrm{CSF})$ macrophages were measured upon coculture with ALI-PBEC and epithelial wounding. a Expression levels of CAMP and TGFB1 were measured in LPS-activated M(GM-CSF) (red bars) or M(M-CSF) (blue bars) macrophages in monoculture or upon coculture with ALI-PBEC ( 3 independent buffy donors). The role of these mediators in epithelial wound closure was assessed by

\section{Macrophage-Derived Mediators Enhance Epithelial Wound Repair}

To determine which macrophage-derived mediators contributed to the observed enhanced epithelial wound repair, we measured the macrophage expression of various growth factors (TGFB1, HGF, IL10, and PDGFA), the metalloproteinase $M M P 9$, and the antimicrobial peptide $C A M P$, all of which have been implicated in epithelial wound repair [5] (Fig. 3a, b). Activated M(M-CSF) macrophages showed higher expression of IL10, and to a lesser extent HGF compared to activated M(GM-CSF) macrophages. Macrophage expression of TGFB1, MMP9, and $P D G F$ was not altered (Fig. 3a, b). Interestingly, LPSactivated M(GM-CSF) macrophages showed increased CAMP (encodes LL-37, an antimicrobial peptide with immune-modulating and wound repair-enhancing activities) expression only upon coculture with ALI-PBEC (Fig. 3a). As CAMP expression in M(GM-CSF) macrophages was increased in presence of LPS and concomitant coculture with ALI-PBEC, we investigated whether this increased CAMP expression may contribute to the observed enhanced wound closure in ALI-PBEC. We therefore added LL-37 and/or TGF- $\beta 1$ (which is known to contribute to epithelial repair) in wounded ALI-PBEC (Fig. 3a). Neither LL-37 nor TGF- $\beta 1$ alone affected wound closure, whereas their combination enhanced wound closure, with complete wound closure at $41 \mathrm{~h}$, as opposed to wound closure at $t=30 \mathrm{~h}$ in the presence of activated M(GM-CSF) macrophages (Fig. 2a). The prolonged time until wound closure upon stimulation with LL-37/ TGF- $\beta 1$, compared to M(GM-CSF) macrophage-induced wound closure, suggests that additional factors in concert with LL-37 and TGF- $\beta 1$ contribute to the observed $\mathrm{M}(\mathrm{GM}-\mathrm{CSF})$ macrophage-enhanced epithelial wound repair.

Therefore, we investigated whether inhibition of the TGF- $\beta$ pathway (SB-431542), matrix metalloproteinases (GM6001), or LL-37 (neutralizing antibody) altered macrophage-induced enhanced epithelial wound repair (online suppl. Fig. 3). Macrophage-induced epithelial wound closure was delayed by both SB-431542 and GM6001.

addition of LL-37 and/or TGF- $\beta 1$ in wounded ALI-PBEC ( 3 independent ALI-PBEC donors). b mRNA expression levels of $H G F$, IL10, MMP9, and PDGFA in M(GM-CSF) (red bars) and M(MCSF) (blue bars) macrophages was measured by qPCR (3 independent buffy donors). Data are shown as mean \pm SEM. ${ }^{*} p<0.05$, ${ }^{* *} p<0.01,{ }^{* * * *} p<0.0001$. ALI-PBEC, primary bronchial epithelial cells cultured at the air-liquid interface; LPS, lipopolysaccharide; $\mathrm{PBEC}$, primary bronchial epithelial cells.

(For figure see next page.)

J Innate Immun 2020;12:410-421 415 


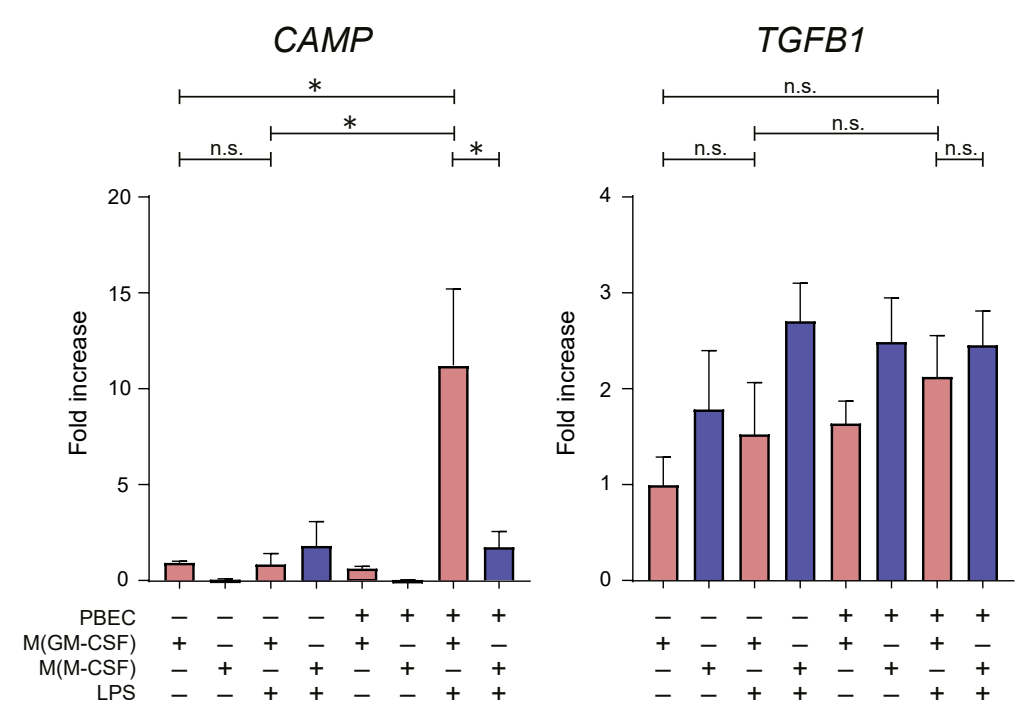

ALI-PBEC wound closure

HGF
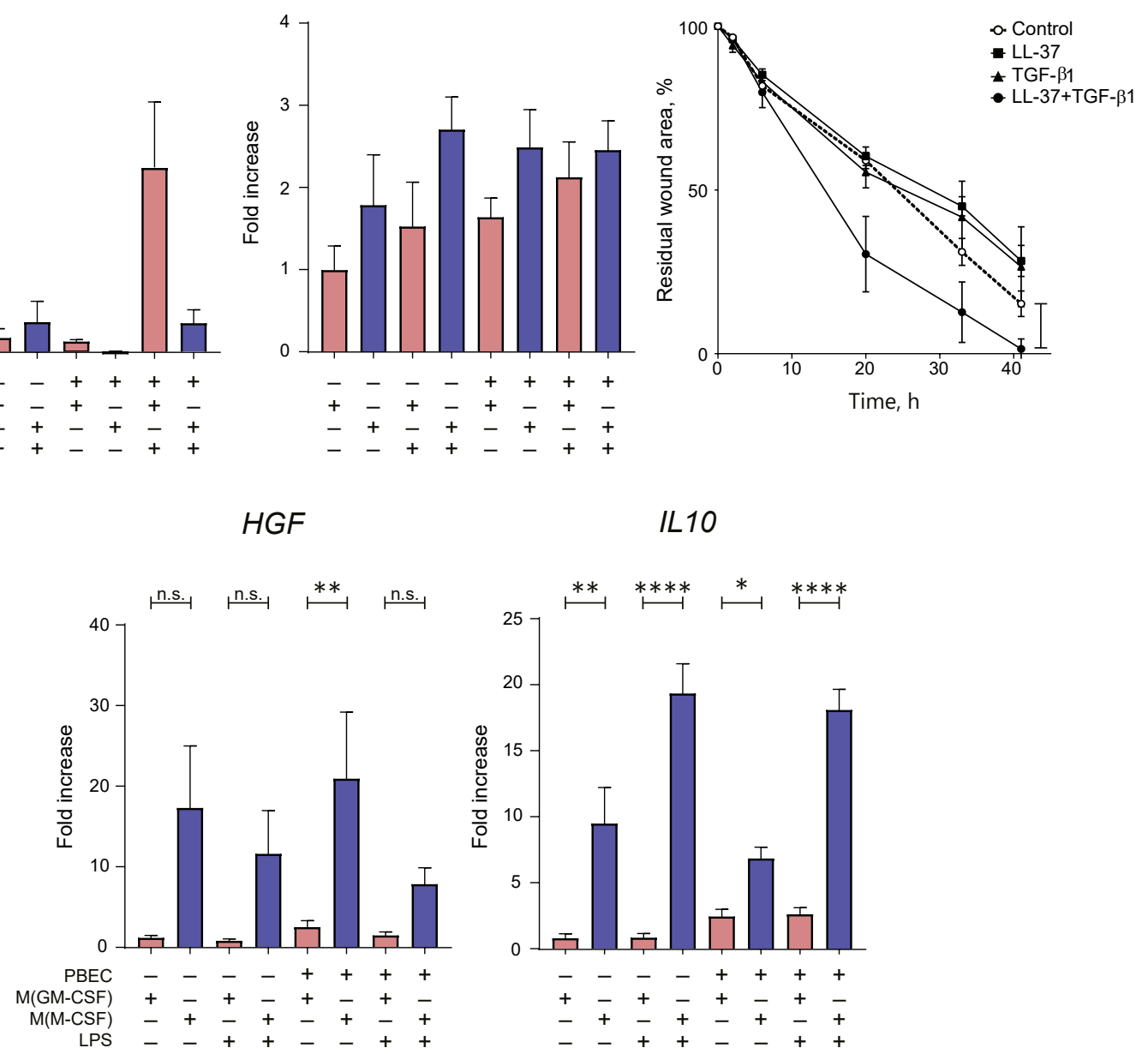

MMP9

PDGFA
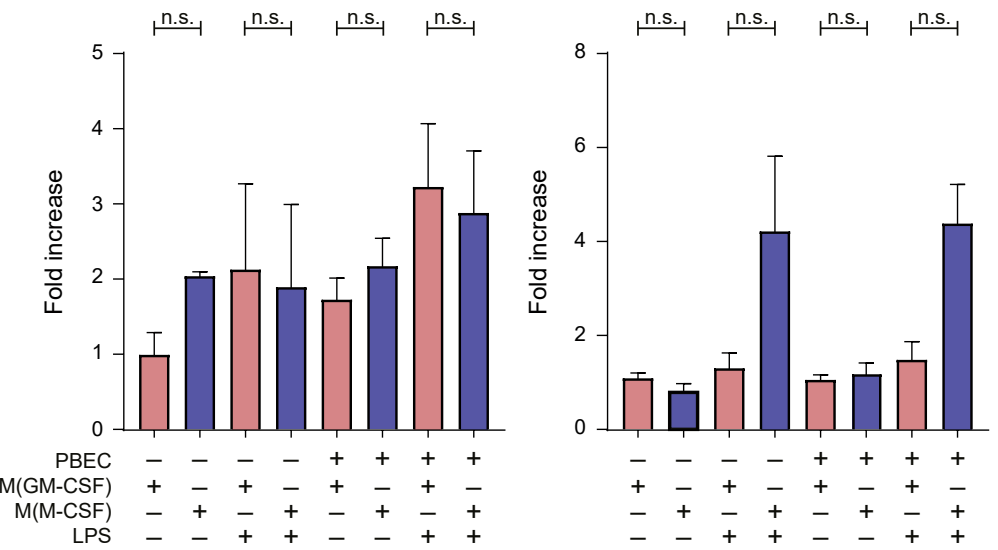

b 
Fig. 4. Effect of ALI-PBEC on CAMP expression in $\mathrm{M}(\mathrm{GM}-\mathrm{CSF})$ macrophages. The mechanisms underlying increased CAMP expression in M(GM-CSF) macrophages in coculture with ALI-PBEC were investigated by exposing macrophages to $\mathrm{CM}$ collected from various culture conditions. a Overview of the culture condition from which the CM was collected (3 independent buffy donors). b CAMP expression at $24 \mathrm{~h}$ was measured by qPCR in $\mathrm{M}$ (GM-CSF) (red bars) or M(M-CSF) macrophages (blue bars) upon stimulation with the various CM. Data are shown as mean \pm SEM. $* p<0.05$. ALI-PBEC, primary bronchial epithelial cells cultured at the air-liquid interface; CM, conditioned medium.

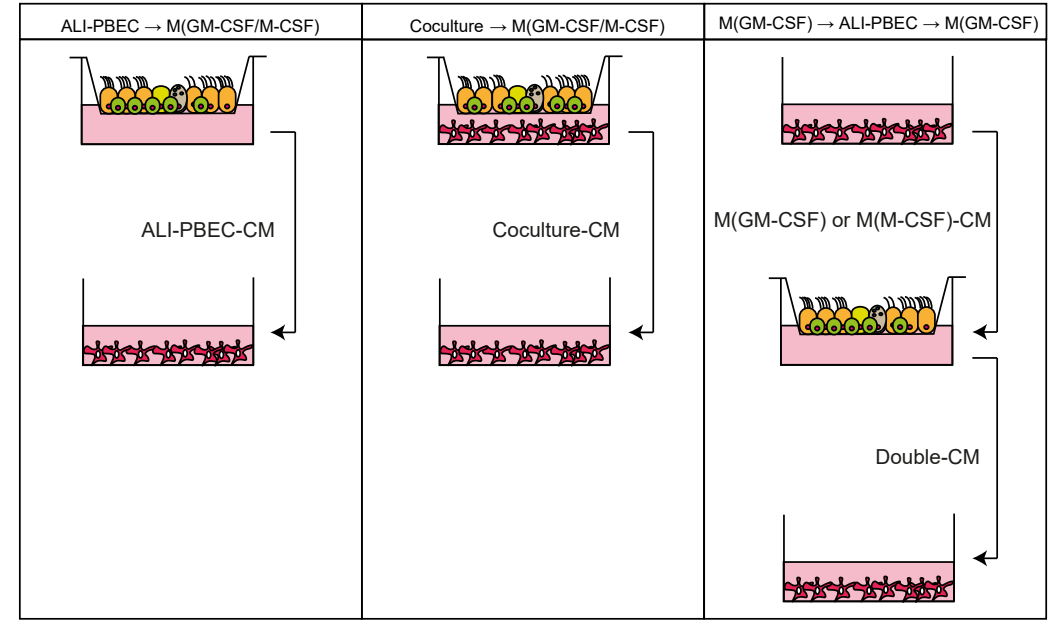

a

CAMP
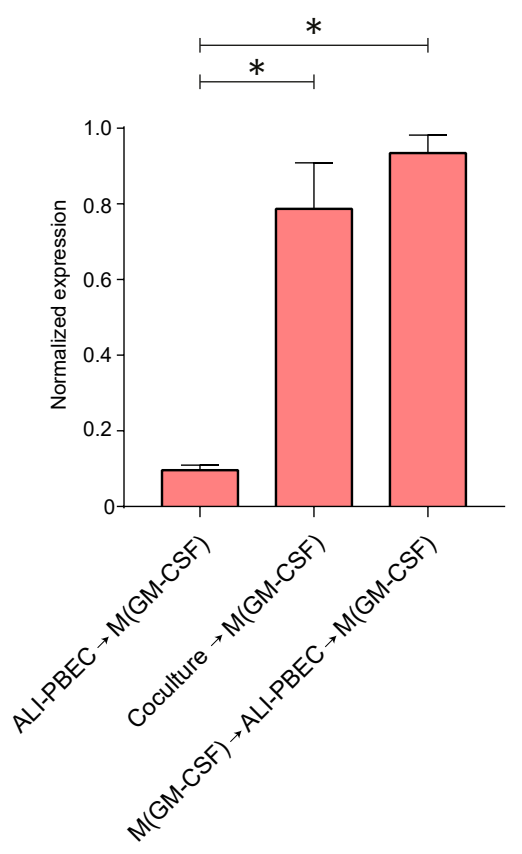

b
CAMP
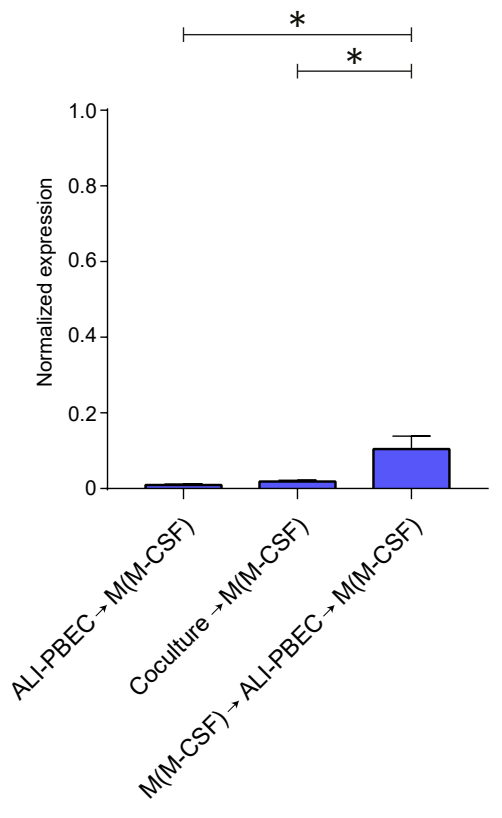

However, since wound closure of ALI-PBEC monocultures was delayed as well in the presence of these compounds, we concluded that the observed delayed wound repair was independent of the presence of macrophages (online suppl. Fig. 3). The contribution of these pathways to macrophage-induced epithelial wound closure therefore remained inconclusive. We studied the contribution of LL-37 to M(GM-CSF)-enhanced epithelial wound re- pair using a selective LL-37-neutralizing antibody; however, this did not affect epithelial wound repair (online suppl. Fig. 3). Collectively, these findings suggest that there is crosstalk between ALI-PBEC and macrophages and that this contributes to epithelial wound repair. We could demonstrate involvement of macrophage-derived factors in enhanced epithelial wound closure, including LL-37 and TGF- $\beta 1$, but also found that crosstalk is not 
restricted to these factors alone. Additionally, $\mathrm{M}(\mathrm{M}-\mathrm{CSF})$ macrophage-derived IL-10, hepatocyte growth factor, and MMP9 are likely candidates as driving factors of $\mathrm{M}(\mathrm{M}-\mathrm{CSF})$-enhanced epithelial wound repair, which we did not further investigate in view of our observation that both types of macrophages enhanced wound repair.

\section{Two-Way Crosstalk between ALI-PBEC and}

$M(G M-C S F)$ Macrophages

We established that activated M(GM-CSF) macrophages increase CAMP expression only in the presence of ALI-PBEC (Fig. 3a). We hypothesized that the increased CAMP in M(GM-CSF) macrophages resulted from crosstalk between the M(GM-CSF) macrophages and epithelial cells. We tested this hypothesis by assessing gene expression in macrophages in experiments using conditioned medium (CM) from ALI-PBEC cultures and epithelial/macrophage cocultures (Fig. 4a). CM was collected from (1) ALI-PBEC only (ALI-PBEC-CM), (2) cocultures of ALI-PBEC and macrophages (Coculture$\mathrm{CM}$ ), and (3) CM that was obtained first from activated macrophages $(24 \mathrm{~h})$ and then added to ALI-PBEC monocultures (24 h) (Double-CM) (Fig. 4a). M(M-CSF) macrophages did not alter CAMP expression upon stimulation with ALI-PBEC-CM, Coculture-CM, or Double-CM (Fig. 4b, blue bars). In contrast, CAMP expression was increased in $\mathrm{M}$ (GM-CSF) macrophages upon addition of Coculture-CM or Double-CM (Fig. 4b, red bars). ALIPBEC-CM was not able to increase CAMP expression in $\mathrm{M}$ (GM-CSF) macrophages, suggesting that CAMP in $\mathrm{M}$ (GM-CSF) macrophages is induced only as a result of a two-way crosstalk between the M(GM-CSF) macrophages and ALI-PBEC.

These data suggest that soluble mediators released by $\mathrm{M}(\mathrm{GM}-\mathrm{CSF})$ macrophages trigger the release of soluble mediators from ALI-PBEC that enhance CAMP expression in $\mathrm{M}$ (GM-CSF) macrophages.

\section{Discussion}

In this study, we investigated the crosstalk between primary ALI-PBEC and polarized macrophages. We found that coculture of well-differentiated ALI-PBEC and activated macrophages displayed interactive crosstalk and influenced epithelial innate immune responses as well as wound repair.

We showed that epithelial IL-6 expression was increased upon coculture with activated M(GM-CSF) and $\mathrm{M}(\mathrm{M}-\mathrm{CSF})$ macrophages compared to epithelial mono- culture. IL-6 is a multifunctional cytokine that has also been shown to promote intestinal epithelial proliferation [21]. Coculture with activated M(GM-CSF) macrophages furthermore increased epithelial expression of DEFB4A and CXCL8. We also confirmed this at the protein level for DEFB4A, as hBD-2 levels in apical washes were increased upon coculture with activated M(GM-CSF) macrophages. The effect on IL-8 secretion was less clear, which may in part be explained by the fact that IL- 8 is produced by epithelial cells as well as by activated macrophages and can be secreted in the basal compartment. hBD-2, however, is produced mainly by the epithelial cells and secreted apically. The induction of hBD-2 in epithelial cells is in line with studies which showed that proinflammatory cytokines induce hBD-2 $[22,23]$ and that microbial products (e.g., LPS) activate macrophages to release inflammatory mediators. Activated proinflammatory macrophages also produce proinflammatory cytokines, including IL- $1 \beta$, that subsequently enhance epithelial production of antimicrobial peptides and inflammatory cytokines [24]. This cascade may act as an amplifying response to microbial products, as ALI-PBEC are less responsive to LPS compared to macrophages [19], which we also found in our study. These findings suggest that activated macrophages alter innate immune responses of well-differentiated ALI-PBEC through the release of soluble mediators.

In addition to an altered epithelial innate immune response, we showed that coculture with activated macrophages significantly enhanced epithelial wound repair. LPS addition to ALI-PBEC alone did not alter wound closure compared to unstimulated control. Our observations are in line with an in vivo response upon epithelial damage: following epithelial damage, proinflammatory macrophages are activated during the inflammatory phase, produce inflammatory cytokines, and display antimicrobial activity [25]. In the subsequent remodeling phase, anti-inflammatory macrophages will contribute to epithelial proliferation and migration followed by further restoration of the epithelial barrier and resolution of inflammation [26].

There was no significant difference between M(GM$\mathrm{CSF})$ and $\mathrm{M}(\mathrm{M}-\mathrm{CSF})$ polarized macrophages in their wound healing capacity. However, M(GM-CSF) polarized macrophages consistently induced faster epithelial wound closure compared to M(M-CSF) polarized macrophages, but this did not reach statistical significance at any of the time points investigated. At present, data on the wound healing capacity of pro- versus anti-inflammatory macrophages are conflicting [27]. Whereas one study 
also showed that M2 macrophage administration was not beneficial in murine cutaneous wound healing [28], this is in contrast with other in vitro studies. These studies showed that anti-inflammatory M(IL-10) macrophages increased the wound repair of A549 epithelial cells compared to proinflammatory $\mathrm{M}(\mathrm{IFN}-\gamma)$ macrophages. This effect may be mediated through IL-10 [24] or hepatocyte growth factor [29], which is a prominent growth factor produced by alveolar macrophages [29] and intestinal macrophages [30]. Also, in our study, hepatocyte growth factor was also expressed in $\mathrm{M}(\mathrm{M}-\mathrm{CSF})$ macrophages and lower in M(GM-CSF) macrophages. Interestingly, IL10 expression was significantly higher in $\mathrm{M}(\mathrm{M}-\mathrm{CSF})$ macrophages compared to $\mathrm{M}(\mathrm{GM}-\mathrm{CSF})$ macrophages, again suggesting that there is cellular crosstalk between ALIPBEC and activated macrophages, although we did not further examine this.

Interestingly, macrophages in bronchoalveolar lavage display higher levels of CD163+ anti-inflammatory macrophages compared to induced sputum [31], suggesting that macrophages in the airways display a proinflammatory phenotype, which may aid in the defense against the heterogeneity of inhaled substances/pathogens. In contrast, M2-type activity of macrophages in the alveolar compartment may protect against excessive inflammation and contributes to repair. This is supported by the proposed role of M2 macrophages in alveolar repair in a mouse pneumonectomy model [32]. In our model, we used ALI-PBEC as a model using epithelial cells isolated from the large conducting airways, where indeed M1 macrophages may induce rapid wound closure to prevent intrusion of harmful pathogens or substances. Depending on localization and microenvironment, the phenotype and function of macrophages may be adapted and thereby influence repair processes [13]. In chronic obstructive pulmonary disease, macrophage polarization has been described to be dysregulated $[33,34]$, which suggests that epithelial wound repair may be affected.

In a previous study we showed that cigarette smoke exposure delayed epithelial wound closure, especially at early time points [14]. We confirmed this in the present study, irrespective of co- or monoculture of ALI-PBEC. However, coculture with both M(GM-CSF) and M(MCSF) macrophages significantly increased epithelial wound closure in the WCS-exposed condition. In our model, only airway epithelial cells were exposed to WCS, whereas macrophages were remained unexposed. Other studies showed that cigarette smoke exposure also affects macrophage function [35], which we did not further investigate.

Airway Epithelial Cell Interaction with Macrophages
M(GM-CSF) macrophages may enhance wound repair in part by the selective increased expression of CAMP upon coculture with ALI-PBEC. We have previously shown that LL-37 drives macrophage polarization towards a proinflammatory macrophage phenotype [36]. Part of the observed effect of M(GM-CSF) macrophages on epithelial innate immunity and wound repair may have been caused by an increased susceptibility of epithelial cells to LPS resulting from exposure to macrophage-derived LL-37 [37]. Apart from its prominent role in host defense, the antimicrobial peptide LL-37 has been shown to be involved in wound repair in both in vivo and in vitro skin models [38, 39]. LL-37 is able to activate airway epithelial cells through epidermal growth factor receptor transactivation [40], which may contribute to wound repair. In our model, exogenously added LL-37 enhanced wound repair only upon concomitant addition of TGF- $\beta 1$. A possible explanation for the synergistic effect of LL-37 or TGF- $\beta 1$ on wound repair could be an interaction between the putative induction of an epithelial migratory (by TGF- $\beta 1$ ) and proliferatory phenotype (by LL-37). We also determined the contribution of other mediators that may enhance wound repair, but since inhibition of the TGF- $\beta 1$ pathway or metalloproteinases also markedly reduced epithelial wound closure in the ALI-PBEC monocultures, we could not determine the role of these pathways in $\mathrm{M}$ (GM-CSF) or M(M-CSF) macrophage-enhanced epithelial wound repair.

We did not observe alterations in macrophage polarization during coculture (data not shown). These findings suggest that in our experimental setup ALI-PBEC did not produce strong polarizing factors or the time in coculture was insufficient to influence macrophage polarization. In this study we focused on only two subsets of macrophages [15], but for future studies our coculture setup allows incorporation of various other macrophage subsets to study epithelial-macrophage interaction [4143]. Whereas other studies of airway epithelial cell cocultures either focused solely on the host defense aspect of macrophages or used cell lines [44-47], we used both primary monocyte-derived macrophages and well-differentiated primary airway epithelial cells, which better reflects in vivo responses. Furthermore, other cell types such as structural cells may be incorporated as well to better mimic the in vivo cellular niche. Previous studies have shown that macrophage-derived mediators have an effect on dermal fibroblasts [48], and this may also occur in the lung, assisting in the modulation of epithelial cell function.

J Innate Immun 2020;12:410-421

DOI: $10.1159 / 000506833$ 
Although a limitation of our model is that the cells are cultured in separate compartments and therefore cellcell interactions are excluded [49], we were able to reveal crosstalk between macrophages and epithelial cells and to show that they interact in part through secreted mediators. In recent years, it has been found that cellular crosstalk may occur through extracellular vesicles, and this has also been described for the interaction between epithelial cells and macrophages [50]. Another limitation is that we did not extend the coculture beyond $72 \mathrm{~h}$ to avoid potential problems with different media requirements for PBEC and macrophages. Future studies are necessary to further optimize this model with prolonged coculture time, without affecting ALI-PBEC integrity and/or M(GM-CSF) or M(M-CSF) macrophage polarization. Finally, we used CD14-monocyte-derived $\mathrm{M}(\mathrm{GM}-\mathrm{CSF})$ and $\mathrm{M}$ (M-CSF) macrophages in this study, whereas these may not fully reflect the repertoire of airway, alveolar, and interstitial macrophages in the lung $[11,13]$. The current culture setup with 2 -week differentiated ALI-PBEC limited the use of freshly isolated lungderived macrophages; however, we did mimic the interaction between airway epithelial cells and recruited monocyte-derived macrophages. Furthermore, whereas macrophage phenotype in vivo displays plasticity, we simplified our model by using polarized macrophages, and thereby could investigate macrophage subtype-specific responses on airway epithelial innate immunity and repair.

In summary, using primary cells, we showed that ALIPBEC and activated macrophage coculture alters epithelial innate immune responses, enhances epithelial wound repair, and induces interactive crosstalk between epithelial cells and macrophages, thereby better representing the in vivo situation compared to monocultures of airway epithelial cells.

\section{Acknowledgements}

We thank Marloes Hofstee and Bram van der Linden (Department of Pulmonology, Leiden University Medical Center, Leiden, The Netherlands) for technical support during this study, and Dr. Anne van der Does (Department of Pulmonology, Leiden University Medical Center, Leiden, The Netherlands) for critically reviewing the manuscript.

\section{Statement of Ethics}

The use of lung tissue for research following surgery within the framework of patient care was in line with the "Human Tissue and Medical Research: Code of conduct for responsible use" [2011] (www.federa.org) that describes the no-objection system for coded anonymous further use of such tissue.

\section{Disclosure Statement}

Dr. de Vos and Dr. Vandeghinste are employees of Galapagos NV. Prof. Hiemstra reports grants from Galapagos NV, grants from the Lung Foundation Netherlands during the conduct of the study, and grants from Boehringer Ingelheim outside the submitted work.

\section{Funding Sources}

This study was supported by grants from the Lung Foundation Netherlands (6.1.14.010 and 6.1.14.009) and Galapagos NV.

\section{Author Contributions}

S. van Riet designed, performed, and analyzed the experiments, interpreted the data, prepared the figures, and drafted the manuscript. A. van Schadewijk provided technical support and helped with data acquisition. S. de Vos and N. Vandeghinste provided input during experimental design and manuscript preparation. J. Stolk, R.J. Rottier, P.S. Hiemstra, and P. Khedoe designed the study and supervised experiments and manuscript writing. All authors read and approved the final version of the manuscript.

\section{References}

1 Hiemstra PS, McCray PB Jr, Bals R. The innate immune function of airway epithelial cells in inflammatory lung disease. Eur Respir J. 2015 Apr;45(4):1150-62.

2 Whitsett JA, Alenghat T. Respiratory epithelial cells orchestrate pulmonary innate immunity. Nat Immunol. 2015 Jan;16(1): 27-35.

3 Hiemstra PS, Amatngalim GD, van der Does AM, Taube C. Antimicrobial Peptides and Innate Lung Defenses: Role in Infectious and Noninfectious Lung Diseases and Therapeutic Applications. Chest. 2016 Feb;149(2):54551.
4 Amatngalim GD, Hiemstra PS. Airway Epithelial Cell Function and Respiratory Host Defense in Chronic Obstructive Pulmonary Disease. Chin Med J (Engl). 2018 May;131(9): 1099-107.

5 Gardner A, Borthwick LA, Fisher AJ. Lung epithelial wound healing in health and disease. Expert Rev Respir Med. 2010 Oct;4(5): 647-60.

6 Alber A, Howie SE, Wallace WA, Hirani N. The role of macrophages in healing the wounded lung. Int J Exp Pathol. 2012 Aug; 93(4):243-51.
7 Gordon S, Martinez-Pomares L. Physiological roles of macrophages. Pflugers Arch. 2017 Apr;469(3-4):365-74.

8 Snyder RJ, Lantis J, Kirsner RS, Shah V, Molyneaux M, Carter MJ. Macrophages: A review of their role in wound healing and their therapeutic use. Wound Repair Regen. 2016 Jul;24(4):613-29.

9 Arora S, Dev K, Agarwal B, Das P, Syed MA Macrophages: their role, activation and polarization in pulmonary diseases. Immunobiology. 2018 Apr-May;223(4-5):383-96.

10 Murray PJ. Macrophage Polarization. Annu Rev Physiol. 2017 Feb;79(1):541-66. 
11 Byrne AJ, Mathie SA, Gregory LG, Lloyd CM. Pulmonary macrophages: key players in the innate defence of the airways. Thorax. 2015 Dec;70(12):1189-96.

$12 \mathrm{Hu} \mathrm{G}$, Christman JW. Editorial: Alveolar Macrophages in Lung Inflammation and Resolution. Front Immunol. 2019 Sep;10:2275.

13 Puttur F, Gregory LG, Lloyd CM. Airway macrophages as the guardians of tissue repair in the lung. Immunol Cell Biol. 2019 Mar; 97(3):246-57.

14 Amatngalim GD, Broekman W, Daniel NM, van der Vlugt LE, van Schadewijk A, Taube C, et al. Cigarette Smoke Modulates Repair and Innate Immunity following Injury to Airway Epithelial Cells. PLoS One. 2016 Nov; 11(11): 0166255.

15 Van't Wout EF, van Schadewijk A, Lomas DA, Stolk J, Marciniak SJ, Hiemstra PS. Function of monocytes and monocyte-derived macrophages in al-antitrypsin deficiency. Eur Respir J. 2015 Feb;45(2):365-76.

16 Amatngalim GD, Schrumpf JA, Dishchekenian F, Mertens TC, Ninaber DK, van der Linden AC, et al. Aberrant epithelial differentiation by cigarette smoke dysregulates respiratory host defence. Eur Respir J. 2018 Apr;51(4):1701009.

17 Andersen CL, Jensen JL, Ørntoft TF. Normalization of real-time quantitative reverse transcription-PCR data: a model-based variance estimation approach to identify genes suited for normalization, applied to bladder and colon cancer data sets. Cancer Res. 2004 Aug;64(15):5245-50.

18 Amatngalim GD, Schrumpf JA, Henic A, Dronkers E, Verhoosel RM, Ordonez SR, et al. Antibacterial Defense of Human Airway Epithelial Cells from Chronic Obstructive Pulmonary Disease Patients Induced by Acute Exposure to Nontypeable Haemophilus influenzae: Modulation by Cigarette Smoke. J Innate Immun. 2017;9(4):359-74.

19 Jia HP, Kline JN, Penisten A, Apicella MA, Gioannini TL, Weiss J, et al. Endotoxin responsiveness of human airway epithelia is limited by low expression of MD-2. Am J Physiol Lung Cell Mol Physiol. 2004 Aug; 287(2):L428-37.

20 Luppi F, Aarbiou J, van Wetering S, Rahman I, de Boer WI, Rabe KF, et al. Effects of cigarette smoke condensate on proliferation and wound closure of bronchial epithelial cells in vitro: role of glutathione. Respir Res. 2005 Nov;6(1): 140 .

21 Kuhn KA, Manieri NA, Liu TC, Stappenbeck TS. IL-6 stimulates intestinal epithelial proliferation and repair after injury. PLoS One. 2014 Dec;9(12):e114195.

22 Kao CY, Chen Y, Thai P, Wachi S, Huang F, Kim C, et al. IL-17 markedly up-regulates betadefensin-2 expression in human airway epithelium via JAK and NF-kappaB signaling pathways. I Immunol. 2004 Sep;173(5):3482-91.

23 O'Neil DA, Porter EM, Elewaut D, Anderson GM, Eckmann L, Ganz T, et al. Expression and regulation of the human beta-defensins hBD-1 and hBD-2 in intestinal epithelium. J Immunol. 1999 Dec;163(12):6718-24.
24 Bals R, Hiemstra PS. Innate immunity in the lung: how epithelial cells fight against respiratory pathogens. Eur Respir J. 2004 Feb;23(2): 327-33.

25 Yamada M, Fujino N, Ichinose M. Inflammatory responses in the initiation of lung repair and regeneration: their role in stimulating lung resident stem cells. Inflamm Regen. 2016 Sep;36(1):15.

26 Smigiel KS, Parks WC. Macrophages, Wound Healing, and Fibrosis: recent Insights. Curr Rheumatol Rep. 2018 Mar;20(4):17.

27 Wynn TA, Vannella KM. Macrophages in Tissue Repair, Regeneration, and Fibrosis. Immunity. 2016 Mar;44(3):450-62.

28 Jetten N, Roumans N, Gijbels MJ, Romano A, Post MJ, de Winther MP, et al. Wound administration of M2-polarized macrophages does not improve murine cutaneous healing responses. PLoS One. 2014 Jul;9(7):e102994.

29 Garnier M, Gibelin A, Mailleux AA, Leçon V, Hurtado-Nedelec M, Laschet J, et al. Macrophage Polarization Favors Epithelial Repair During Acute Respiratory Distress Syndrome. Crit Care Med. 2018 Jul;46(7):e692-701.

30 D’Angelo F, Bernasconi E, Schäfer M, Moyat M, Michetti P, Maillard MH, et al. Macrophages promote epithelial repair through hepatocyte growth factor secretion. Clin Exp Immunol. 2013 Oct;174(1):60-72.

31 Kunz LI, Lapperre TS, Snoeck-Stroband JB, Budulac SE, Timens W, van Wijngaarden S, et al.; Groningen Leiden Universities Corticosteroids in Obstructive Lung Disease Study Group. Smoking status and anti-inflammatory macrophages in bronchoalveolar lavage and induced sputum in COPD. Respir Res. 2011 Mar;12(1):34.

32 Lechner AJ, Driver IH, Lee J, Conroy CM, Nagle A, Locksley RM, et al. Recruited monocytes and type 2 immunity promote lung regeneration following pneumonectomy. Cell Stem Cell. 2017 Jul;21(1):120-34.e7.

33 Hiemstra PS. Altered macrophage function in chronic obstructive pulmonary disease. Ann Am Thorac Soc. 2013 Dec;10 Suppl:S180-5.

34 Shaykhiev R, Krause A, Salit J, Strulovici-Barel Y, Harvey BG, O'Connor TP, et al. Smokingdependent reprogramming of alveolar macrophage polarization: implication for pathogenesis of chronic obstructive pulmonary disease. J Immunol. 2009 Aug;183(4):2867-83.

35 Strzelak A, Ratajczak A, Adamiec A, Feleszko W. Tobacco Smoke Induces and Alters Immune Responses in the Lung Triggering Inflammation, Allergy, Asthma and Other Lung Diseases: A Mechanistic Review. Int J Environ Res Public Health. 2018 May;15(5):E1033.

36 van der Does AM, Beekhuizen H, Ravensbergen B, Vos T, Ottenhoff TH, van Dissel JT, et al. LL-37 directs macrophage differentiation toward macrophages with a proinflammatory signature. J Immunol. 2010 Aug;185(3):1442-9.

37 Shaykhiev R, Beisswenger C, Kändler K, Senske J, Püchner A, Damm T, et al. Human endogenous antibiotic LL-37 stimulates airway epithelial cell proliferation and wound clo- sure. Am J Physiol Lung Cell Mol Physiol. 2005 Nov;289(5):L842-8.

38 Ramos R, Silva JP, Rodrigues AC, Costa R, Guardão L, Schmitt F, et al. Wound healing activity of the human antimicrobial peptide LL37. Peptides. 2011 Jul;32(7):1469-76.

39 Carretero M, Escámez MJ, García M, Duarte B, Holguín A, Retamosa L, et al. In vitro and in vivo wound healing-promoting activities of human cathelicidin LL-37. J Invest Dermatol. 2008 Jan; 128(1):223-36.

40 Tjabringa GS, Aarbiou J, Ninaber DK, Drijfhout JW, Sørensen OE, Borregaard N, et al. The antimicrobial peptide LL-37 activates innate immunity at the airway epithelial surface by transactivation of the epidermal growth factor receptor. J Immunol. 2003 Dec;171(12):6690-6.

41 Gindele JA, Mang S, Pairet N, Christ I, Gantner F, Schymeinsky J, et al. Opposing effects of in vitro differentiated macrophages sub-type on epithelial wound healing. PLoS One. 2017 Sep;12(9):e0184386.

42 Boyette LB, Macedo C, Hadi K, Elinoff BD, Walters JT, Ramaswami B, et al. Phenotype, function, and differentiation potential of human monocyte subsets. PLoS One. 2017 Apr; 12(4):e0176460.

43 Murray PJ, Wynn TA. Protective and pathogenic functions of macrophage subsets. Nat Rev Immunol. 2011 Oct;11(11):723-37.

44 Bodet C, Chandad F, Grenier D. Inflammatory responses of a macrophage/epithelial cell co-culture model to mono and mixed infections with Porphyromonas gingivalis, Treponema denticola, and Tannerella forsythia. Microbes Infect. 2006 Jan;8(1):27-35.

45 Blom RA, Erni ST, Krempaská K, Schaerer O, van Dijk RM, Amacker M, et al. A Triple CoCulture Model of the Human Respiratory Tract to Study Immune-Modulatory Effects of Liposomes and Virosomes. PLoS One. 2016 Sep;11(9):e0163539.

46 Bauer RN, Müller L, Brighton LE, Duncan $\mathrm{KE}$, Jaspers I. Interaction with epithelial cells modifies airway macrophage response to ozone. Am J Respir Cell Mol Biol. 2015 Mar; 52(3):285-94.

47 Reuschl AK, Edwards MR, Parker R, Connell DW, Hoang L, Halliday A, et al. Innate activation of human primary epithelial cells broadens the host response to Mycobacterium tuberculosis in the airways. PLoS Pathog. 2017 Sep;13(9):e1006577.

48 Ploeger DT, Hosper NA, Schipper M, Koerts JA, de Rond S, Bank RA. Cell plasticity in wound healing: paracrine factors of M1/M2 polarized macrophages influence the phenotypical state of dermal fibroblasts. Cell Commun Signal. 2013 Apr;11(1):29.

49 Leoni G, Neumann PA, Sumagin R, Denning TL, Nusrat A. Wound repair: role of immuneepithelial interactions. Mucosal Immunol. 2015 Sep;8(5):959-68

50 Lee H, Abston E, Zhang D, Rai A, Jin Y. Extracellular Vesicle: An Emerging Mediator of Intercellular Crosstalk in Lung Inflammation and Injury. Front Immunol. 2018 Apr;9:924. 